Annals of the Rheumatic Diseases, 1988; 47, 1027-1030

Case report

\title{
Intra-articular apatite deposition in mixed connective tissue disease: crystallographic and technetium scanning characteristics
}

\author{
C W HUTTON, ${ }^{1}$ P J MADDISON, ${ }^{1}$ A J COLlins, ${ }^{2}$ AND J A BERRIMAN \\ From the ${ }^{1}$ Royal National Hospital for Rheumatic Diseases, Bath; and the Departments of ${ }^{2}$ Pharmacy and \\ Pharmacology and ${ }^{3}$ Material Science, Bath University
}

SUMMARY An acute arthritis in a patient with mixed connective tissue disease (MCTD) was found to be associated with intra-articular deposition of carbonated hydroxyapatite crystals. A technetium hydroxymethylene diphosphonate bone scan showed intense uptake in the delayed phase scan of the affected joints. Synovial fluid analysis demonstrated uptake of the radiopharmaceutical drug directly onto the crystals.

Key words: hydroxyapatite, calcinosis.

\section{Case report}

A 35 year old woman presented with an acutely painful swollen erythematous right index metacarpophalangeal joint. A similar acute arthritis had developed in the metacarpophalangeal joint of the right middle finger two months previously. This had settled spontaneously over two weeks. She had a seven year history of a peripheral polyarthritis affecting the small joints of her hands and wrists and associated with marked flexion contractures and sclerodactyly. She had severe Raynaud's phenomenon. Two years previously she had had an acute episode of vasculitis and pericarditis. She was not systemically unwell with the acute arthritis, which settled on treatment with non-steroidal antiinflammatory drugs over three weeks.

Radiology of the hands before the episode of the acute arthritis showed an erosive arthritis primarily affecting the interphalangeal joints and the first carpometacarpal joints. Periarticular calcification was present, particularly around the ring and little finger proximal interphalangeal joints. Radiographs

Accepted for publication 29 April 1988.

Correspondence to Dr C W Hutton, Royal National Hospital for Rheumatic Diseases, Upper Borough Wall, Bath BA1 1RL. at the time of the acute arthritis showed extensive opacification around the index and middle metacarpophalangeal joints of the right hand (Fig. 1).

A technetium- $99 \mathrm{~m}$ four hour delayed phase scan showed increased activity over a number of interphalangeal, metacarpophalangeal, and wrist joints. The most intensive activity was over the index and middle metacarpophalangeal joints of the right hand (Fig. 2).

Analysis of synovial fluid from both the acutely affected joints showed large numbers of nonbirefringent crystals. .ere were no associated inflammatory cells. Scanning electron microscopy showed plate-like crystals characteristic of apatite. Electron diffraction of both crystal powder and individual crystals showed patterns identical to those obtained from a pure hydroxyapatite sample supplied by Bio-Rad (Fig. 3). Infrared spectroscopy showed a pattern characteristic of carbonated hydroxyapatites. Analysis of the radioactivity of the synovial fluid after centrifugation showed intense activity in the pellet, that was not lost in washing, and no activity in the supernatant, demonstrating that the technetium had bound to the crystals forming the pellet.

Serological investigation showed the presence of high titre anti-U1/ribonucleoprotein antibodies 


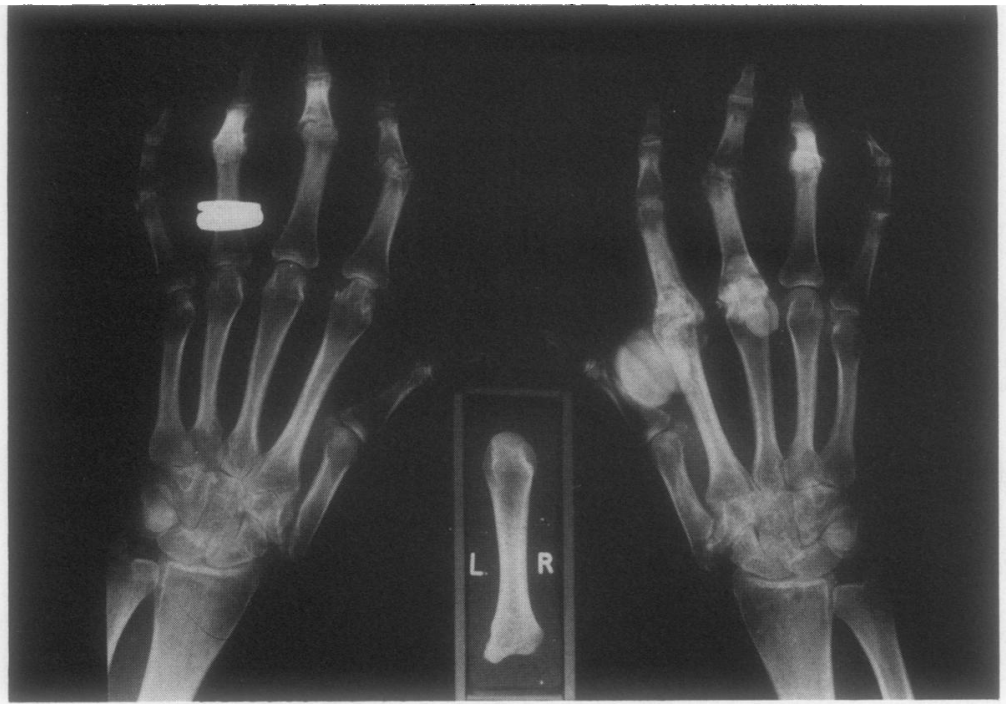

Fig. 1 Radiograph of the hand showing erosive arthritis involving the first carpometacarpal, interphalangeal, कै and metacarpal joints, periarticular dystrophic calcification around the proximal interphalangeal joints of the left little ring fingers, and loculated synovial fluid calcification of the index and middle right metacarpophalangeal joints.

(1/256), positive antinuclear antibodies (>640 IU), with a speckled pattern of immunofluorescence. Rheumatoid factor and anti-Sm antibodies were not detected. Immunoglobulin, complement, and renal function were normal. Serum calcium and phosphate and parathyroid hormone were also normal.

\section{Discussion}

This case shows that an acute arthritis associated with apatite deposition may occur in mixed connective tissue disease (MCTD). This widens the spec- $N$ trum of arthritis seen in this condition. Initially a $N$ non-erosive symmetrical arthritis was described as characteristic. ${ }^{1}$ Later an erosive arthritis was ${ }^{\mathrm{\omega}}$ reported. ${ }^{2}$ This latter pattern appears to be very? similar to the chronic erosive arthritis seen in this case. Synovial calcification in MCTD is rare, however. ${ }^{2} 3$

Intra-articular calcification appears to be particu- $\frac{T}{0}$ larly unusual, with only one case that is probably $\underset{\mathbb{D}}{\mathrm{D}}$ 


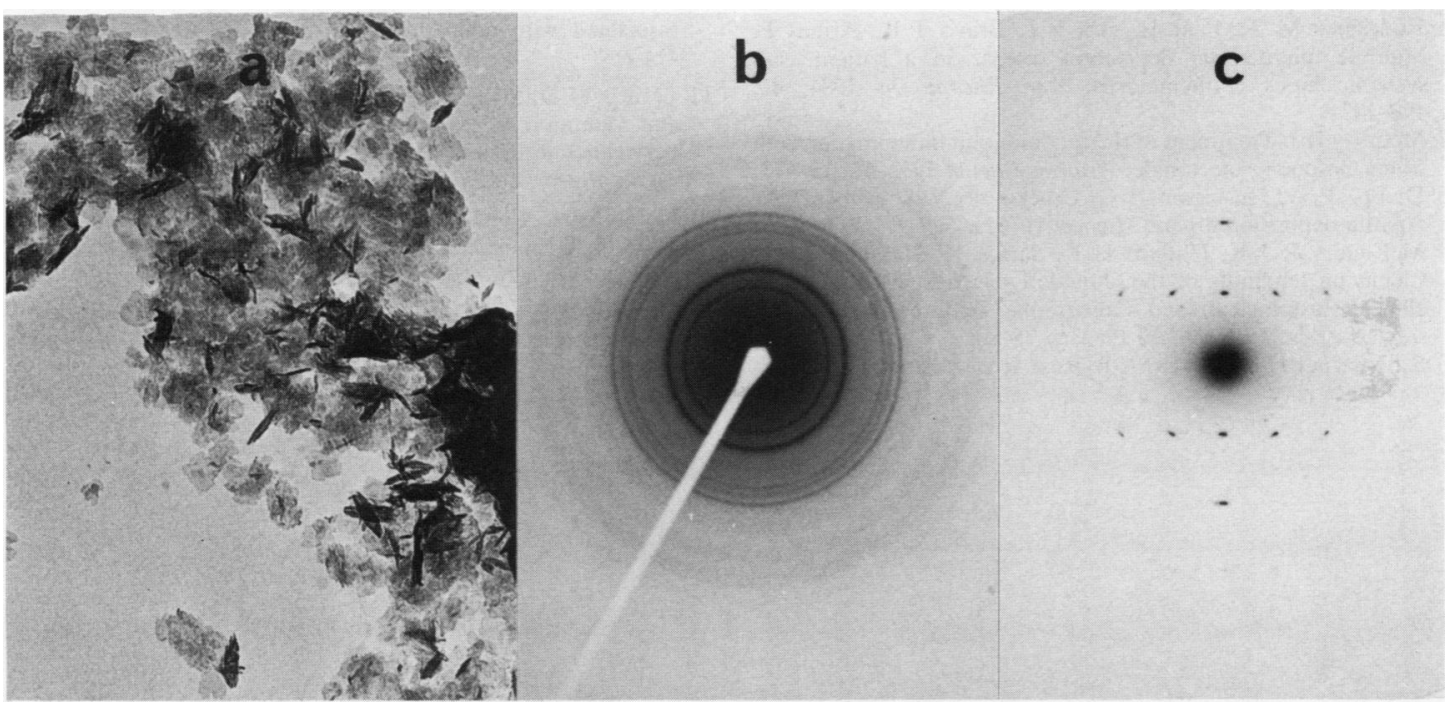

Fig. 3 (a) Electron micrographs of particles from the right index metacarpophalangeal joint $(1 \mathrm{~cm}=225 \mathrm{~nm})$. $(b, c)$ Electron diffraction patterns of joint apatite crystal powder and of a single joint crystal $(1 \mathrm{~cm}=3.4 \AA$ on $B r a g g$ spacing).

MCTD showing a loculated pattern of calcification very similar to that seen in this case, in association with a chronic synovitis. ${ }^{4}$ The MCTD syndrome has many features of systemic lupus erythematosus, scleroderma, and rheumatoid arthritis, and in all these conditions periarticular calcification is seen. It may possibly be precipitated by intra-articular steroid injections. ${ }^{5-7}$ Why the deposition occurs remains uncertain, but it may reflect abnormalities in the connective tissue, defective crystal inhibition, or solute characteristics.

Apatite deposition occurs in a number of other conditions, including calcific periarthritis and osteoarthritis. ${ }^{89}$ Although apatite deposition can produce experimental inflammation in vivo, its role in the inflammatory reaction seen in apatite associated conditions remains uncertain. It may be deposited as a consequence of inflammation. ${ }^{10}$ In this patient the apatite deposition appears to indicate that a process different from the chronic erosive synovitis has occurred.

The delayed bone scan imaged the joints with an erosive arthritis, but the most intensive activity was at the metacarpophalangeal joints where the crystal deposition had occurred. The finding of uptake onto the crystals indicates this activity is due to direct uptake in the joint fluid. Technetium hydroxymethylene diphosphonate bone scan localisation has been demonstrated in several ways. In experimental systems these include absorption onto the crystals and onto connective tissue proteins such as newly synthesised collagen. ${ }^{11} 12$ The synovial fluid analysis here shows that direct absorption onto crystals is important in man.

Crystal deposition may occur in connective tissue diseases like MCTD more frequently than has previously been recognised. In systemic lupus erythematosus monosodium urate deposition is now recognised to occur more often than would be expected by chance. ${ }^{13}$ Possibly, apatite deposition is more common than has been recognised as a cause of acute arthritis in MCTD. In an acute arthritis with no evidence of infection analysis of the synovial fluid for crystals may allow a more precise diagnosis and avoid unnecessary treatment, as well as giving greater insight into the aetiology of the arthritis.

\section{References}

1 Sharp G C, Irwin W S, Tan E M, Gould R G, Holman H R. Mixed connective tissue disease-an apparently distinct rheumatic disease syndrome associated with a specific antibody to extractable nuclear antigen (ENA). Am J Med 1972; 52: 148-58.

2 Ramos-Niembro F, Alarco-Segovia D, Hernandez-Ortiz J. Articular manifestations of mixed connective tissue disease. Arthritis Rheum 1979; 22: 43-51.

3 Bennet R M, O'Connell D J. The arthritis of mixed connective tissue disease. Ann Rheum Dis 1978; 37: 397-403.

4 Reginato A J, Schumacher H R. Synovial calcification in a patient with collagen vascular disease: light and electron microscopic studies. J Rheumatol 1977; 4: 261-71.

5 Resnick D, Scavulli J F, Goergen T G, Genant H K, Niwayama G. Intra-articular calcification in scleroderma. Radiology 1977; 124: $685-8$. 
6 Rodriguez M A, Paul H, Abadi I, Bravo J R, Armas P. Multiple microcrystal deposition disease in a patient with systemic lupus erythematosus. Ann Rheum Dis 1984; 43: 498-502.

7 McCarty D J. Treatment of rheumatoid joint inflammation with triamcinolone hexacetonide. Arthritis Rheum 1972; 15: 157-73.

8 Dieppe P A, Huskisson E C, Crocker P, Willoughby D A. Apatite deposition disease. Lancet 1976; i: 266-9.

9 McKendry R J R, Uhthoff H K, Sarkar K, Hyslop P St G. Calcifying tendinitis of the shoulder: prognostic value of the clinical, histological, and radiographic features in surgically treated cases. J Rheumatol 1982; 9: 75-80.

10 Schumacher H R, Smolyo A P, Rose R L, Maurer K. Arthritis associated with apatite crystals. Ann Intern Med 1977; 87: 414-6.

11 Francis M D, Slough C L, Tofe A J. Factors affecting uptake and retention of technetium-99m-diphosphonate and $99 \mathrm{~m}$ pertechnetate in osseous connective and soft tissues. Calcified Tissue Research 1976; 20: 303-11.

12 Rosenthall L, Kaye M. Observations on the mechanisms of $99 \mathrm{~m}$ Tc-labelled phosphate complex uptake in metabolic bone disease. Semin Nucl Med 1976; 6: 59-67.

13 Greenfield D I, Fong J S, Barth W F. Systemic lupus is erythematosus and gout. Semin Arthritis Rheum 1985; 14: 176-9. 